\title{
Effect of Ocular Deviation on Stereopsis
}

\author{
Partha Haradhan Chowdhury ${ }^{1^{*}}$, Brinda Haren Shah ${ }^{2}$ \\ ${ }^{1}$ M.Optom, Department of Optometry, Shree Satchandi Jankalyan Samiti Netra Prasikshan Sansthan, Pauri, Affiliated to \\ Uttarakhand State Medical Faculty, Dehradun, India \\ ${ }^{2}$ M.Optom, Department of Optometry, Gujarat University, Gujarat, India
}

Received: 21 May, 2018; Accepted: 06 June, 2018; Published: 18 June, 2018

*Corresponding author: Partha Haradhan Chowdhury, M. Optom, Department of Optometry, Shree Satchandi Jankalyan Samiti Netra Prasikshan Sansthan, Pauri, Affiliated to Uttarakhand State Medical Faculty, Dehradun, India, E-mail: optometrypublish@gmail.com

\begin{abstract}
Purpose: The aim of the present study is to evaluate Stereopsis in the presence of ocular deviation
\end{abstract}

Methods: A pilot, cross sectional, observational study was performed at tertiary eye care centers. Subjects with ocular deviation between 10 to 40 prism diopters, corrected distance Visual Acuity should be greater than $6 / 18$ and Age should be between 10 to 40 years of age were included in the study. Stereopsis was evaluated using the Titmus Fly Test. Mean scores of visual parameters were taken.

Results: A total of 30 subjects were included in the study. Of these $53 \%$ of subjects were in the age group of $11-20$ years, $40 \%$ subjects were in the age group of $21-30$ years and $7 \%$ subjects were in the age group of $31-40$ years. $60 \%$ subjects were Female. Stereopsis was deteriorated to 80 secs of arc in the presence of ocular deviation.

Conclusions: In cases of ocular deviation, stereopsis deteriorates significantly.

Keywords: Ocular Deviation; Stereopsis;

\section{Introduction}

Stereopsis is the third grade of Binocular Single vision. Grades of Binocular Single vision are measured by Synaptophore [1]. Stereopsis occurs due to disparity of an object. When an object is placed on the horopter, some part of the object is placed in front of the horopter and some part is placed behind the horopter and due to its disparity within the range, patient will develop Depth Perception [2]. It is not always mandatory that Stereopsis is always associated with three dimensional objects [3]. It also occurs when two dimensional objects is offset horizontally. Due to this offset depth perception is created. Pannum Fusional Area is considered as an in front and behind the horopter to some extent $[4,5]$. It is steeper centrally and flatter peripherally. So, in case of ocular deviation, images of an object are displaced from the foveal region to parafoveal region and due to cone cell variation stereoscopic range will also be varied [6,7].

\section{Methodology}

A pilot, cross sectional, observational study was performed at tertiary eye care centers. Written\& Oral consent was taken by each and every subject. Subjects with Ocular deviation between 10 to 40 prism diopters, Corrected distance Visual Acuity should be greater than 6/18 and Age group of 10 years to 40 years were included in the study. Individuals with any other systemic disease(specially which can affect study), Individuals with any other Ocular Pathology, with any active ocular infection, any ocular anomalies like corneal scar etc ,ocular deviation if less than 10 degree and Significant amount of amblyopic patient were excluded from the study. Full refractive correction along with detailed fund us evaluation was performed in each and every patient. Stereopsis was measured with Titmus Fly Test. Statistical Data was analyzed using SPSS software version 20 and Microsoft Office Excel 10.

\section{Results}

30 subjects were included in the study. (Graph 1) shows distribution of subjects in various age groups. 53\% of subjects were in the age group of $11-20$ years, $40 \%$ of subjects were in

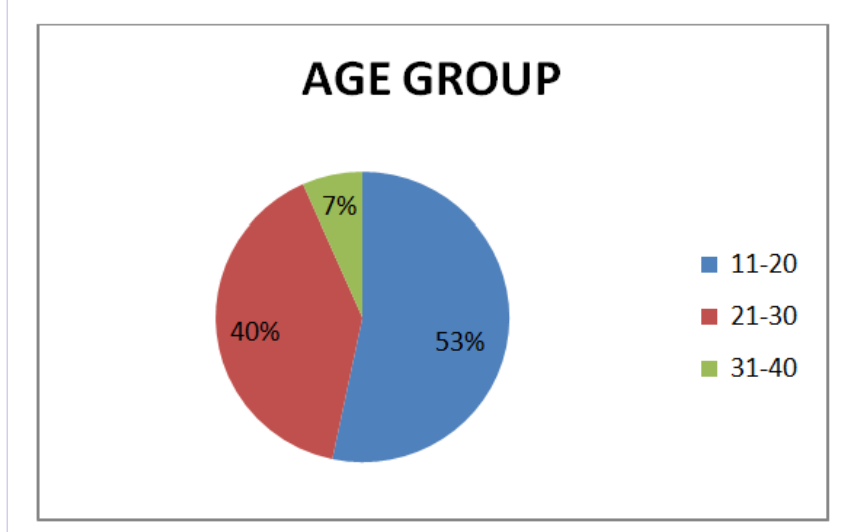

Graph 1:Shows Age Wise Distribution of the Subject

the age group of 21-30 years and subjects were in the age group of 31-40 years. (Graph 2) shows gender wise distribution of the subjects. $60 \%$ subjects were Female. Mean scores of visual parameters were taken using SPSS Software version 20. (Graph 3) shows mean Stereopsis was deteriorated to 80 secs of arc. 


\section{Discussion}

et al.

Normal value of Stereopsis is 40 secs of arc when recorded with Titmus Fly test. According to statistical analysis, it is shown that in cases of ocular deviation, Mean Stereopsis was deteriorated to 80 secs of arc. According to the studies, it has been proved that with increase in ocular deviation, stereoscopic ranges will be varied. It mainly occurs due to the fact that images are shifted from the foveal to parafoveal region and due to number of cone cell deterioration from foveal region to parafoveal region.

\section{Conclusion}

Stereopsis decreases with increase in ocular deviation

\section{References}

1. Wright KW, |Spiegel PH, Thompson L. Handbook of Pediatric Strabismus and Amblyopia. First Ed, 2006.

Graph 2: Shows gender wise distribution of the subjects

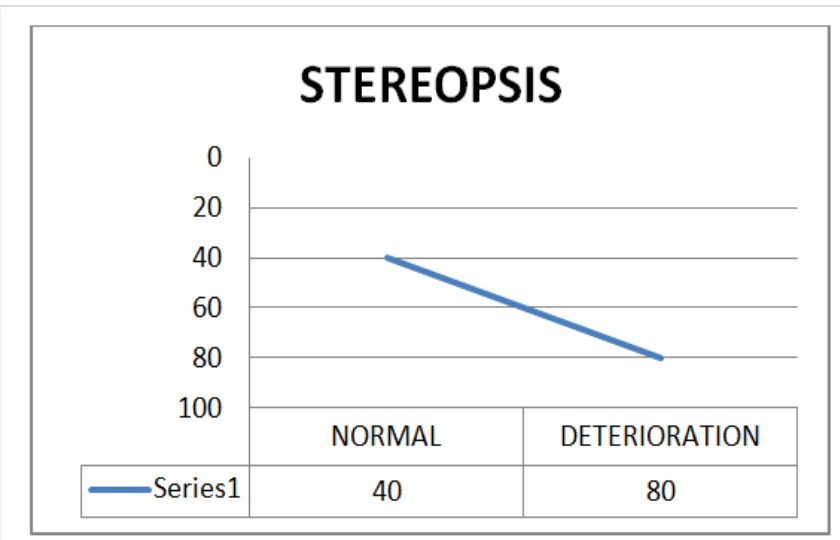

Graph 3: Shows comparison of mean Stereopsis to the normal subjects
2. Hui Zhu, Jia-Jia Yu, Rong-Bin Yu, Hui Ding, Jing Bai, Ji Chen, et al. Association between Childhood Strabismus and Refractive Error in Chinese Preschool Children. PLoS One. 2015;10(3):e0120720.

3. Rajavi Z, Sabbaghi H, Baghini AS, Yaseri M, Sheibani K, Norouzi G. Prevalence of Color Vision Deficiency and its Correlation with Amblyopia and Refractive Errors among Primary School Children. J Ophthalmic Vis Res. 2015;10(2):130-138.

4. Tandon AK, Velez FG, Isenberg SJ, Demer JL, Pineles SL. Binocular Inhibition in Strabismic Patients is Associated with Diminished Quality of Life. J AAPOS. 2014;18(5):423-426.

5. Ye XC, Pegado V, Patel MS, Wasserman WW. Strabismus genetics across a spectrum of eye misalignment disorders. Clin Genet. 2014;86(2):103-111.

6. Koçak-Altintas AG, Satana B, Koçak I, Duman S. Visual Acuity and Color Vision deficiency in Amblyopia. Eur J Ophthalmol. 2000;10(1):77-81.

7. Freeman AW, Nguyen VA, Jolly N. Components of Visual Acuity Loss in Strabismus. Vision Res. 1996;36(5):765-774. 